\title{
Ausschreibungen
}

Philip Wilbrandt

\section{AO Fellowships}

Die AO-Stiftung bietet verschiedene Fellowships für unterschiedliche Zielgruppen an. Die Teilnahmebedingungen unterscheiden sich, abgesehen von der Mitgliedschaft in der AOTrauma, die für alle Voraussetzung ist.

Weitere Informationen zu allen Fellowship-Programmen finden Sie unter:

https://www.aofoundation.org/Structure/education/ fellowships/Pages/fellowship.aspx

\section{AOTrauma Fellowships 2018}

Jedes Jahr fördert die AOTrauma International über 250 Fellowships weltweit. Aktuell gibt es über 120 AO-Kliniken auf 5 Kontinenten, in denen die Fellowships absolviert werden können.

Es gibt 2 unterschiedliche Fellowship-Programme:

1. „Visit the Expert Fellow“ ist für erfahrene Chirurgen, die zur Verbesserung eines ganz speziellen fachlichen Gebiets für 2-4 Wochen zu einem entsprechenden Experten reisen wollen. Hier gibt es keine Altersbeschränkung.

2. „AOTrauma Fellow“ ist für jüngere Ärzte bis maximal 45 Jahre. Dauer 4-12 Wochen.

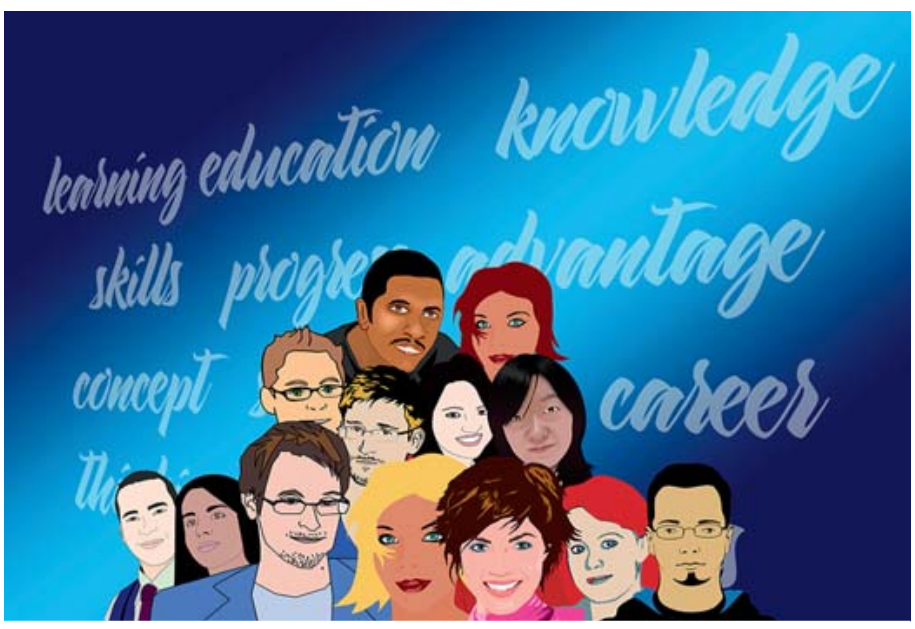

- Abb. 1 Fellowships.
Grundvoraussetzung sind die Absolvierung des AO-Prinzipien- und AO-Fortgeschrittenenkurses (bis Antritt des Fellowships).

Der Bewerbungsschluss für die weltweiten AOTrauma Fellowships für das Jahr 2018 ist am 14. Juli 2017.

Weitere Informationen finden Sie unter www.aotrauma. org/fellowship

\section{CID Clinical Research Fellowship für Ärzte 2018}

Seit 2005 haben 33 Ärzte ein Fellowship bei AO Clinical Investigation und Documentation (CID) absolviert. Diese 3-monatigen Fellowships ermöglichen Ärzten, an klinischer Forschung, insbesondere Studien, mitzuwirken und dabei konkrete Kenntnisse im Bereich der Studienplanung, Monitoring, Datenanalyse/-auswertung und der Publikation zu erwerben.

Bewerbungen sind einzureichen bis zum 05.09.2017.

Ansprechperson ist Frau Anahí Hurtado, Tel.: + 41/44/ 200-2471, E-Mail: anahi.hurtado@aofoundation.org

\section{ARI Medical Research Fellowship 2019}

Dieses Fellowship ermöglicht Ärztinnen/Ärzten am AOForschungsinstitut (ARI) in der Schweiz Erfahrungen in der angewandten Forschung zu sammeln. Die ausgewählte Person arbeitet an einem konkreten Forschungsprojekt mit und wird im Erfolgsfall Koautor/-in.

Das Fellowship dauert 12 Monate, in Ausnahmefällen 6. Das Stipendium beinhaltet die finanzielle Unterstützung der Aufenthaltskosten. In der Bewerbung sollte konkret benannt werden, in welchem der Forschungsfelder des ARIs das Interesse an einer Mitarbeitet besteht.

Die Bewerbungsdeadline ist 30. September 2017. Die Entscheidungen werden Anfang 2018 bekannt gegeben. Ansprechperson ist Frau Sonia Wahl, Tel.: + 41/81/4142541, E-Mail: sonia.wahl@aofoundation.org 


\section{AOTrauma Deutschland Forschungsnachwuchs}

Die AOTrauma Deutschland (AOTD) unterstützt 2-mal jährlich Forschungsprojekte mit insgesamt 75000 Euro. Damit möchte die AOTD den wissenschaftlichen Nachwuchs im klinischen und experimentellen Bereich mit einer Anschubfinanzierung sowie einem Mentoring fördern. Die nächste Ausschreibungsfrist endet am 31. August 2017.

Bewerben können sich klinisch aktive Unfallchirurgen, die nicht älter als 35 Jahre sind und in Deutschland an Universitätskliniken sowie regionalen und kommunalen Krankenhäusern tätig sind. Eingereicht werden können sowohl klinische als auch experimentelle Forschungskonzepte aus den Bereichen Traumatologie, Mund-Kiefer-Gesichts-Chirurgie und Veterinärchirurgie (nicht Wirbelsäule). Es werden nur Bewerbungen von Mitgliedern der deutschen AO angenommen, deren Forschungsarbeit von der jeweiligen Klinikleitung unterstützt wird. Die Anträge (bitte nicht größer als $10 \mathrm{MB}$ ) sind bis zum 31. August 2017 zu senden an:

Prof. Dr. Georg Duda

Julius-Wolff-Institut

Augustenburger Platz 1, D-13353 Berlin

E-Mail: georg.duda@charite.de

Aus den eingegangenen Bewerbungen wählt die AOTD 5 Beiträge aus und lädt die Antragsteller ein, ihre Arbeiten auf der Auswahlsitzung während der Jahrestagung der DGOU am 26. Oktober 2017 in Berlin vorzustellen und zu verteidigen. Bis zu 3 der Kandidaten werden dann mit einer Anschubfinanzierung von maximal 12000 Euro ausgezeichnet. Die Anwesenheit in Berlin ist Voraussetzung zur Teilnahme.

\section{AOTrauma-Wissenschaftspreis 2017}

Der Wissenschaftspreis der AOTrauma Deutschland (AOTD) wird jährlich vergeben. Die Preisverleihung findet am 25. Oktober 2017 auf dem AOTD-Symposium im Rahmen des DKOU statt, auf dem die Arbeit präsentiert werden darf. Das Preisgeld beträgt $7500 €$. Für den diesjährigen Preis werden noch Bewerbungen entgegengenommen.

Zu erfüllende Kriterien sind:

- Das Thema der Arbeit muss im Tätigkeitsbereich der AOTrauma, AOCMF oder AOVET liegen.

- Es können sowohl klinische Forschungsarbeiten, Grundlagenforschung, systematische Reviews als auch Metaanalysen eingereicht werden.

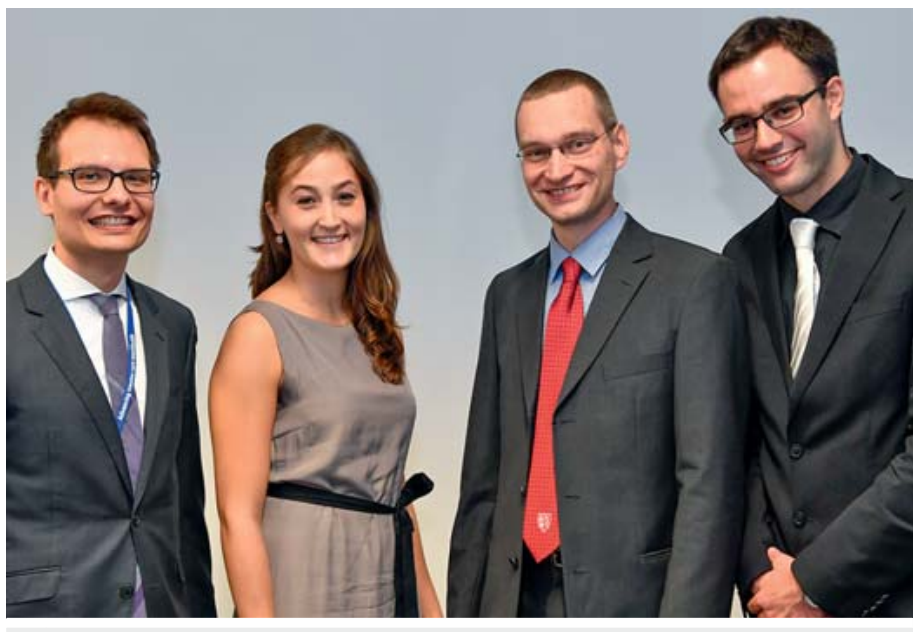

- Abb. 2 Die Prämierten der Nachwuchsförderung: Verboket, Goffart, Zwingenberger und Orth. Bild: Jürgen Staiger.

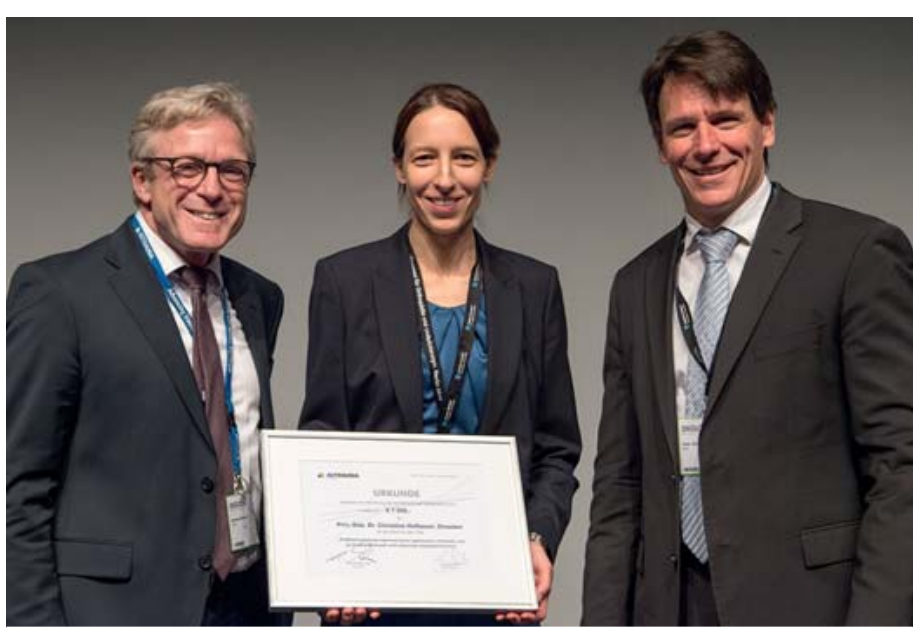

- Abb. 3 Präsident M. Raschke, Wissenschaftspreisträgerin Christine Hofbauer und Research Officer G. Duda. Bild: Jürgen Staiger.

- Die Arbeit muss bereits in einem Peer-reviewed Journal publiziert sein.

- Der/die Bewerber/-in muss AOTrauma-, AOCMF- oder AOVET-Mitglied sein.

- Der/die Preisträger/-in muss bei der Preisverleihung am 25.10.2017 in Berlin anwesend sein.

- Einzureichen sind: die Arbeit, ein Motivationsbrief und der Lebenslauf.

Eine Auswahlkommission wird in einem anonymisierten Auswahlverfahren die Entscheidung treffen.

Einsendungen müssen bis zum 31.08.2017 eingegangen sein bei:

Prof. Dr. Georg Duda

Julius-Wolff-Institut 


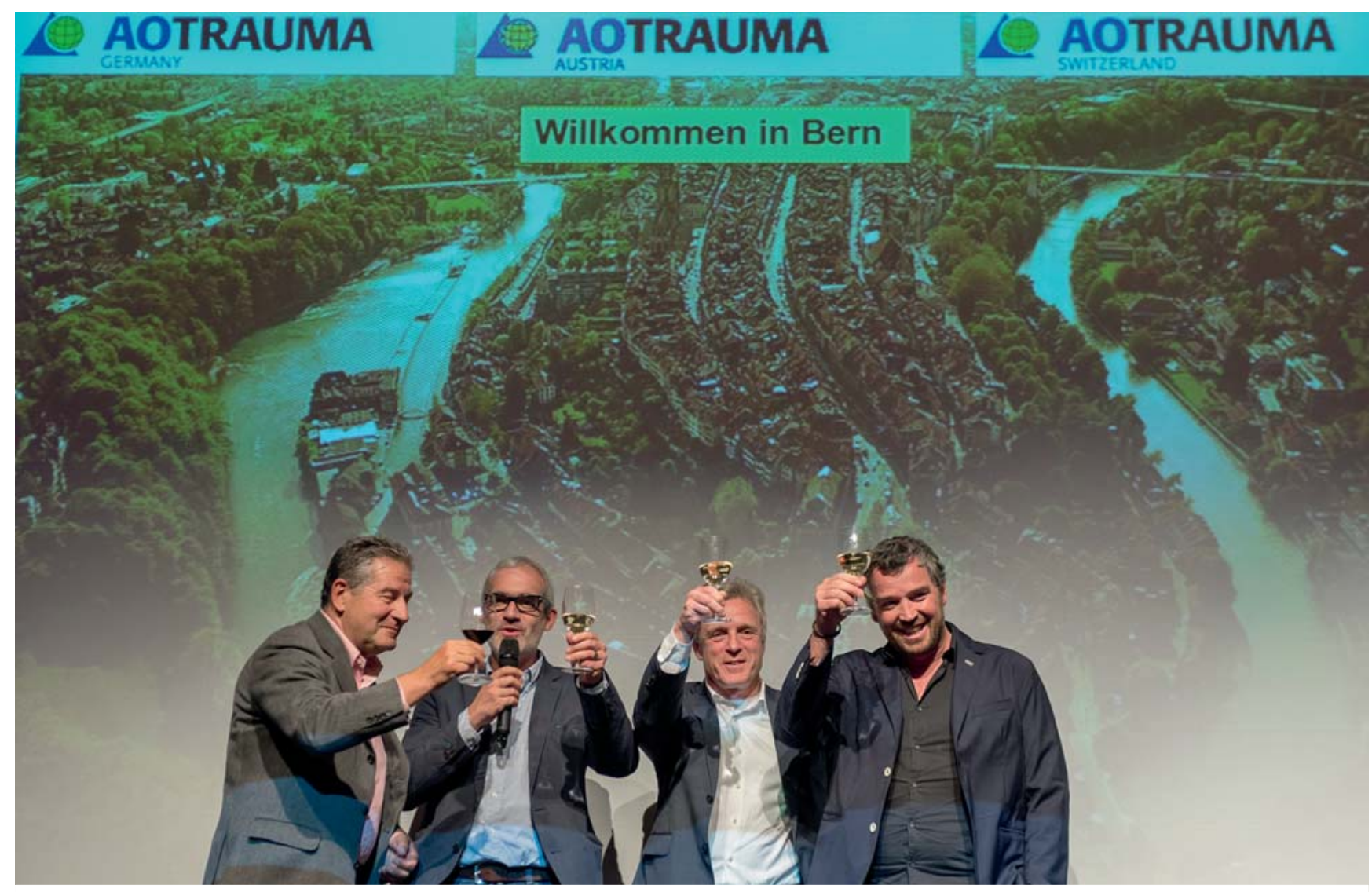

- Abb. 4 Wagner (Präsident A), Keel (Gastgeber 3-Länder-Tagung 2016 in Bern), Raschke (Präsident D) und Borens (Präsident CH). Bild: Jürgen Staiger.

Augustenburger Platz 1, D-13353 Berlin

E-Mail: georg.duda@charite.de

\section{D-A-CH-Reisestipendium 2017}

Die 3 AO-Sektionen von Deutschland (D), Österreich (A) und der Schweiz $(\mathrm{CH})$, abgekürzt D-A-CH, schreiben jeweils ein Reisestipendium aus. Ziel ist es, den Austausch von klinisch aktiven Ärzten zwischen den 3 Ländern zu fördern. Bewerbungen müssen als Zielland eines der beiden Partnerländer haben.

\section{Konzept}

- Vorgesehen sind 14-21 Tage Aufenthalt in einer AOKlinik im D-A-CH-Bereich.

- Die Fördersumme pro Stipendium beträgt $1500 €$.

- Insgesamt gibt es 3 Stipendien pro Jahr.

- Jedes Land vergibt 1 Stipendium.

\section{Bewerbungskriterien}

- klinisch aktive/r Ärztin/Arzt

- AOTrauma-Mitgliedschaft im Land, wo der Arzt/die Ärztin tätig ist
- Einreichung eines Erfahrungsberichts nach dem Aufenthalt

\section{Einzureichende Bewerbungsunterlagen sind}

- Motivationsschreiben (max. 1 Seite) mit Angabe der Zielklinik

- CV

- Nachweis der AO-Mitgliedschaft

Bewerbungen für das Stipendium der AOTrauma Deutschland (also von in Deutschland tätigen Ärzten) können bis Sonntag, 2. Juli 2017 per E-Mail eingereicht werden an Philip Wilbrandt: p.wilbrandt@aodeutschland.de

Bibliografie

DOI https://doi.org/10.1055/s-0043-109278

OP-JOURNAL 2017; 33: 90-92 @ Georg Thieme Verlag KG Stuttgart · New York ISSN 0178-1715 